\title{
A Method for the Estimation of the Square Size in the Chessboard Image using Gray-level Co-occurrence Matrix
}

\author{
Guan $\mathrm{Xu}^{1,2}$, Xiaotao $\mathrm{Li}^{2, *}$, Jian $\mathrm{Su}^{1}$, Rong $\mathrm{Chen}^{1}$, Jianfang $\mathrm{Liu}^{2}$ \\ ${ }^{1}$ Department of Vehicle Application Engineering, Traffic and Transportation College, Nanling Campus, Jilin University, \\ Renmin Street 5988\#, 130025, Changchun, Jilin, P. R. China, xuguan@jlu.edu.cn \\ ${ }^{2}$ Mechanical Science and Engineering College, Nanling Campus, Jilin University, Renmin Street 5988\#, 130025, \\ Changchun, Jilin, P. R. China, *lixiaotao@jlu.edu.cn
}

\begin{abstract}
The paper proposes a new simple procedure for measuring the square size employing the gray-level co-occurrence matrix of a chessboard image. As the size of the square structure in a chessboard image provides the geometric constraint information among the corners, it is available to improve the precision of extracting corners and serve the camera calibration. The co-occurrence matrix of a chessboard image is constructed to obtain the statistic information of the grayscale distribution. The $2 \mathrm{D}$ offset of the matrix is parameterized to calculate the correlation which is regarded as the implication of the repetition probability of the similar textures. A descending tendency is observed in the experiments because the similarity decreases with the greater offset. However, minimum and maximum are captured in the correlation curve, which represents that the square texture reappears with the periods of one and two square size, separately. The size of the square is tested by applying the first minimum of the correlation. The experiments are performed on the horizontal and vertical directions which are corresponding to the length and the width of the square, respectively. The experiments prove that the described method has the potential to measure square size of the chessboard.
\end{abstract}

Keywords: Chessboard image, measurement, gray-level co-occurrence matrix, correlation

\section{INTRODUCTION}

Oalt HESSBOARD IMAGE which comprises squares with alternating black and white squares is a typical analyzed object in vision-based measurement. It contains much information corresponding to deriving the space position of an observed target. Therefore it is important to measure the square size of a chessboard in different scale images precisely for understanding and analyzing the objects.

A number of heuristic rules in accordance with respective characteristics of the images have been developed for the research fields of parts manufacturing, visual system calibration, feature segmentation and pose estimation, etc. [1]-[5]. There are, for example, increasing applications that require precise calibration of cameras to perform measurements on objects located in images. De la Escalera et al. proposed an automatic algorithm to reduce the consuming time in calibration procedure [1]. The proposed method uses a pattern similar to a chessboard. The calibration is carried out by means of a combined analysis of two Hough transforms, corner detection and invariant properties of the perspective transformation. The fundamental content is to construct the relationship between the camera parameters and the $2 \mathrm{D}$ coordinates of the corners in the chessboard image. The size of the square structure in a chessboard image represents the geometric constraint information among the corners. The distance constraint is available to enhance the coordinate precision of the corners and serve the camera calibration. The former methods for obtaining the size of square depend on the feature recognition and extraction. The first class is based on the region segmentation and contour extraction of the squares on the chessboard, such as Tsai C.H. algorithm, Rosenfeld A. algorithm, Freeman H. algorithm and Xiao J.L. algorithm, etc. [6]-[9]. The other class is calculating the grayscale distribution in the neighborhood of square corner points, such as Lucas-Kanade, SUSAN, SIFT and Harris algorithm, etc. [10]-[13]. The measurement effects rely on the processes of region segmentation or feature extraction.

Real-world engineering problems such as industrial measurement are also expected to be solved by a widely suitable approach. In some cases, as the recognized object is confused by the complex background, the lack of feature information leads to incorrect segmentation and effects the traditional steps of segmentation, object recognition, feature extraction and computation. To separate the square from the image and determine the size of square, the texture characteristic of the chessboard which is expressed by cooccurrence matrix is adopted to recognize and analyze the target board image in the process. The proposed method aims at providing a measurement approach based on grayscale statistic information to identify and decide the square size precisely and efficiently.

\section{PROposed Method}

The chessboard in the image consists of several equal squares which are arranged in two alternating colors, "black" and "white". The grayscale varies periodically in the image which involves a chessboard. Based on the texture feature above, an approach to solve the estimation problem is completed by gray-level co-occurrence matrix of the image. A co-occurrence matrix is a matrix that is defined on a grayscale image to determine the distribution of cooccurring values at a given offset. Mathematically, a cooccurrence matrix $M$ is defined on an $n \times m$ image $I$, with the parameter of an offset $(\Delta x, \Delta y)$, as[14]:

$$
M_{\Delta x, \Delta y}(i, j)=\sum_{p=1}^{n} \sum_{q=1}^{m} \begin{cases}1, & I(p, q)=i \text { and } I(p+\Delta x, q+\Delta y)=j \\ 0, & \text { otherwise }\end{cases}
$$


where $(p, q)$ is the pixel coordinate in the image, $(i, j)$ is the coordinate of an element in the co-occurrence matrix $M .(i, j)$ also stands for the grayscale value of the specified pixel in the original position $(p, q)$ and the other pixel value with an offset $(\Delta x, \Delta y)$ in the image. The offsets $\Delta x, \Delta y$ vary from zero to a half of the image width which is equal to 300 pixels in the experiments. The step of the offsets $\Delta x, \Delta y$ is one pixel. If the grayscale in $(p, q)$ of the image is equal to $i$ while the grayscale in $(p+\Delta x, q+\Delta y)$ is equal to $j$, the element value in $(i, j)$ of the co-occurrence matrix plus one, is iterated.

For industrial purposes, a detailed and precise measurement of the square size is necessary. Correlation is one of the statistics specified in properties of the gray-level co-occurrence matrix. It is expressed by [14]

$$
C(\Delta x, \Delta y)=\sum_{i}^{n} \sum_{j}^{m} \frac{\left(i-\mu_{i}\right)\left(j-\mu_{j}\right) M_{\Delta x, \Delta y}(i, j)}{\sigma_{i} \sigma_{j}}
$$

where $M(i, j)$ is the element of the co-occurrence matrix $M$ with the coordinate $(i, j), \mu_{i}, \mu_{j}$ are the mathematical expectation values of the elements in the $i$ th row, $j$ th column, $\sigma_{i}, \sigma_{j}$ are the variance values of the elements in row $i$, column $j$. For each co-occurrence matrix vectors of row and column, arithmetic means $\mu_{i}, \mu_{j}$ and standard deviations $\sigma_{i}$, $\sigma_{j}$ are to be calculated.

Considering that the $(\Delta x, \Delta y)$ parameterization conducts the co-occurrence matrix sensitivity to the texture of the chessboard, an offset vector is employed as a variable so that the periodicity of the image texture which is corresponding to the size of black or white squares results in a series of different co-occurrence matrices for the same image. From this point of view, the co-occurrence matrices are formed by a series of offsets sweeping through the half length of an image side to achieve a degree of texture invariance.

In order to investigate the square size of the chessboard image, the correlation values of the co-occurrence matrices are calculated by (1) and (2) while the offset varies in $x, y$ directions, respectively. Then we construct the relationship between the offset and the correlation which is selected to locate the maximum and minimum related to the boundaries of the squares. The length and width of the square over the chessboard are obtained by the boundary information in the image.

\section{EXPERIMENTAL RESULTS AND DISCUSSION}

The presented measurement method has been performed on a Celeron $1.73 \mathrm{GHz}$ notebook computer with $2 \mathrm{~GB}$ memory. The image resolution is $800 \times 600$ pixels.

Two chessboard images, Fig.1(a) and Fig.2(a), in different rotational angles and the other two images, Fig.3(a) and Fig.4(a), in a greater scale are captured in the experiments for evaluating the effects of the method in various cases. The matrices are calculated to the raw chessboard image. Considering the real measurement situation, the size of square is commonly lower than the half length of an image. Therefore, the variable of the offset value covers the reasonable interval from 1 pixel to 300 pixels aiming to find the minimum and maximum related to the boundaries of the squares. One pixel is chosen as the step in the $x$-axis scale of the plots in Fig.1(b)-Fig.4(b) and Fig.1(c)-Fig.4(c). The experiments are performed on the condition that the offset changes in vertical and horizontal directions, individually. Texture correlation as a function of offset in vertical or horizontal directions is illustrated in Fig.1(b)-Fig.4(b) and Fig.1(c)-Fig.4(c). Texture correlation as a function of offsets in vertical direction and horizontal direction is shown in Fig.1(d)-Fig.4(d). The curves briefly show a downward tendency because the correlation is insignificant to the greater offset, which also implies the decreasing repetition probability of similar textures in the increasing distance. However, there are 2 maximum points and 2 minimum points in each curve. The first maximum point stands for the width and length of two squares because the black or white pixels on the target reappear most often in the distance of two squares. The minimum point shows the width and length of a single square, as the black or white pixels infrequently repeat on the target in the distance of a single square. The same results are deduced in the distance of squares with even or odd numbered times, respectively. Based on the discussion above, the first minimum point depicts the size of a square on the chessboard; likewise, the first maximum sketches the repeated patterns of two squares, and yet the third peak is unrecognized in the correlation curve as there is no periodical texture in the image larger than four squares of the chessboard. The selected cooccurrence matrices with the horizontal offsets of one and double squares are presented in Fig.1(e)-Fig.4(e) and Fig.1(f)-Fig.4(f), individually. In Fig.1(e)-Fig.4(e), the higher values of the matrix are grouped close to four areas. For the horizontal offset of one square, the areas near $(0$, $255)$ and $(255,0)$ imply that many black and white pixels of the chessboard repeat in the interval distance of one square. The areas near $(0,0)$ and $(255,255)$ describe that there are also many reappeared black or white pixels in the interval distance of one square. In Fig.1(f)-Fig.4(f), the higher values of matrix integrate to the two areas near $(0,0)$ and $(255,255)$ because the black or white pixels of the chessboard tend to reoccur on the period of two squares. Furthermore, comparing Fig.1(e) to Fig.3(e), and Fig.1(f) to Fig.3(f), the concentration of the matrix values is higher in Fig. 3 as the chessboard texture occupies a larger area of the image.

As the length and the width of a square correspond to the first minimum points of the correlation curves in vertical and horizontal directions, respectively, the computed square width and length in Fig.1(a)-Fig.4(a) are reported in Table1, which satisfy the test results of manual operation within 1 pixel error. Considering the first minimum and maximum corresponding to one and two squares, the offset of the first maximum should be exactly the double of the first minimum. The ratio of offsets on the first maximum and minimum points is calculated to evaluate the method precision in Table2. The mean value of the ratio is 1.933 with the variance 0.004 .

The experiments prove that the measurement approach for the square size of a chessboard image taking the advantage of correlation of gray-level co-occurrence matrix is capable of measuring the length and width in different scale images. 


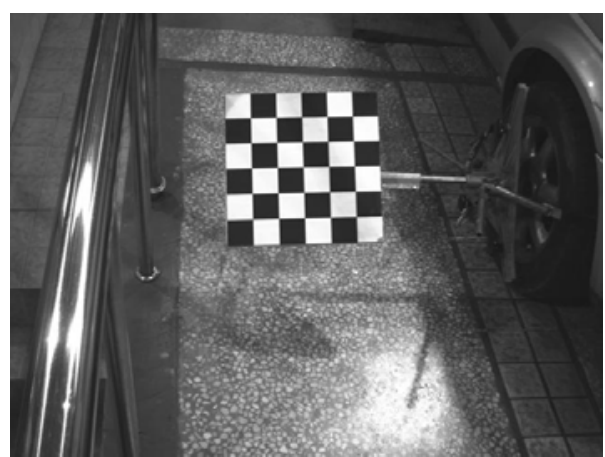

(a)

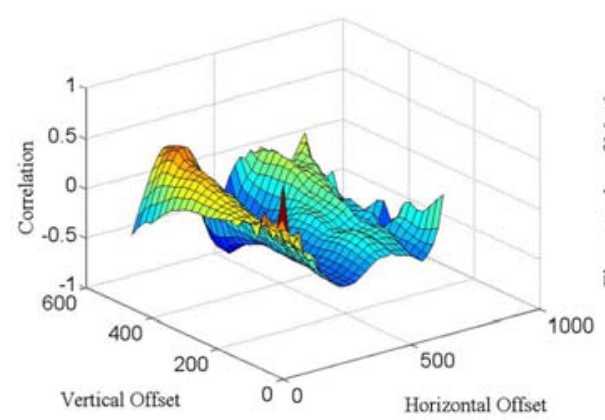

(d)

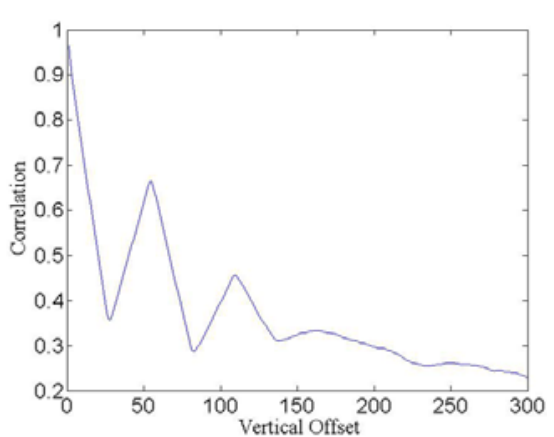

(b)

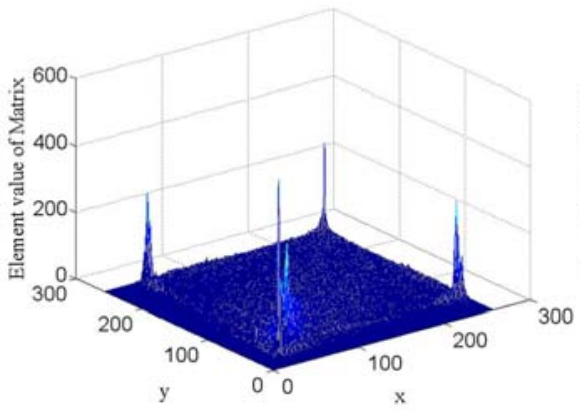

(e)

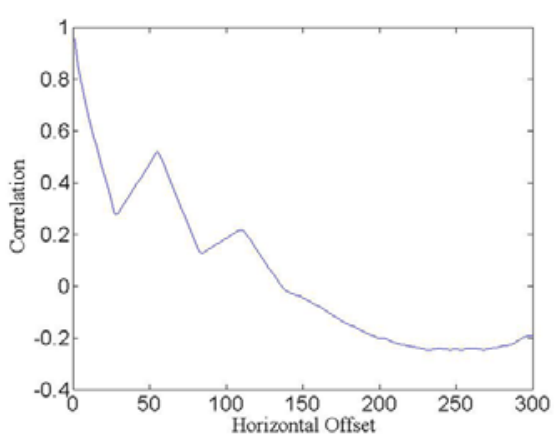

(c)

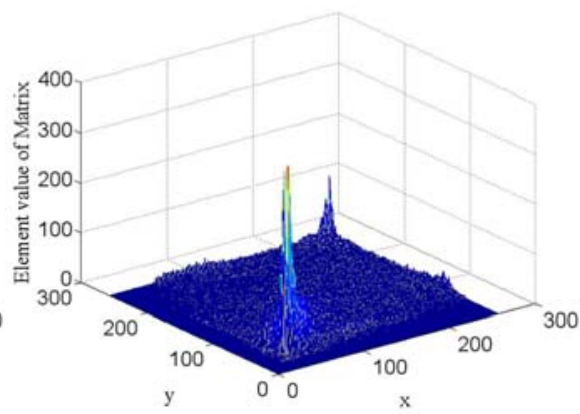

(f)

Fig.1. Experimental results on an orthographic chessboard image. (a) Raw image, (b) Texture correlation as a function of offset in vertical direction, (c) Texture correlation as a function of offset in horizontal direction, (d) Texture correlation as a function of offsets in vertical direction and horizontal direction, (e) Co-occurrence matrix with the horizontal offset of one square, (f) Co-occurrence matrix with the horizontal offset of two squares.

Table 1. Measurement results of the squares in Fig.1(a)-Fig.4(a) (the unit is pixel)

\begin{tabular}{|c|c|c|c|c|}
\hline & Fig.1(a) & Fig.2(a) & Fig.3(a) & Fig.4(a) \\
\hline Computed square width & 28 & 32 & 47 & 52 \\
\hline Counted square width & 27 & 31 & 48 & 51 \\
\hline Computed square length & 28 & 26 & 46 & 43 \\
\hline Counted square length & 27 & 25 & 46 & 42 \\
\hline
\end{tabular}

Table 2. Ratio of the offsets on the first maximum and minimum points in Fig.1(b)-Fig.4(b) and Fig.1(c)-Fig.4(c) (the unit is pixel)

\begin{tabular}{|c|c|c|c|c|c|}
\hline \multicolumn{2}{|c|}{ Offset } & Fig.1(a) & Fig.2(a) & Fig.3(a) & Fig.4(a) \\
\hline \multirow{3}{*}{$\begin{array}{c}\text { Horizontal } \\
\text { offset }\end{array}$} & $\begin{array}{c}\text { Minimum } \\
\text { point }\end{array}$ & 28 & 32 & 47 & 52 \\
\hline & $\begin{array}{c}\text { Maximum } \\
\text { point }\end{array}$ & 55 & 58 & 93 & 101 \\
\hline & $\begin{array}{l}\text { Ratio of } \\
\text { the offsets }\end{array}$ & 1.964 & 1.813 & 1.979 & 1.942 \\
\hline \multirow{3}{*}{$\begin{array}{l}\text { Vertical } \\
\text { offset }\end{array}$} & $\begin{array}{c}\text { Minimum } \\
\text { point }\end{array}$ & 28 & 26 & 46 & 43 \\
\hline & $\begin{array}{c}\text { Maximum } \\
\text { point }\end{array}$ & 55 & 48 & 92 & 84 \\
\hline & $\begin{array}{l}\text { Ratio of } \\
\text { the offsets }\end{array}$ & 1.964 & 1.846 & 2.000 & 1.953 \\
\hline
\end{tabular}




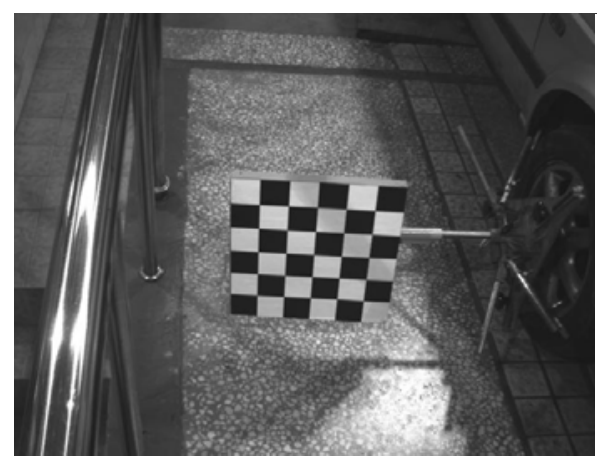

(a)

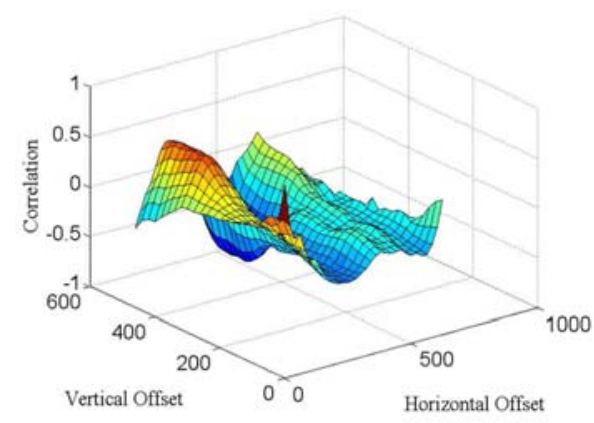

(d)

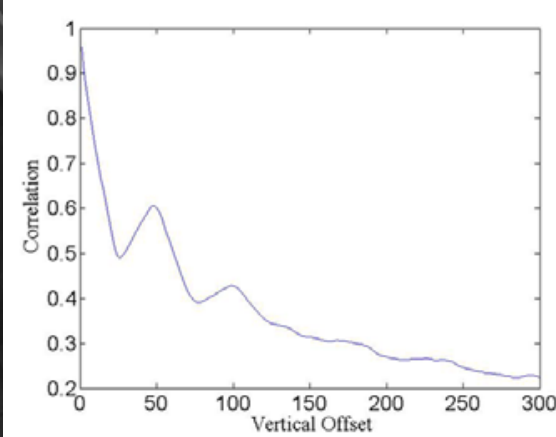

(b)

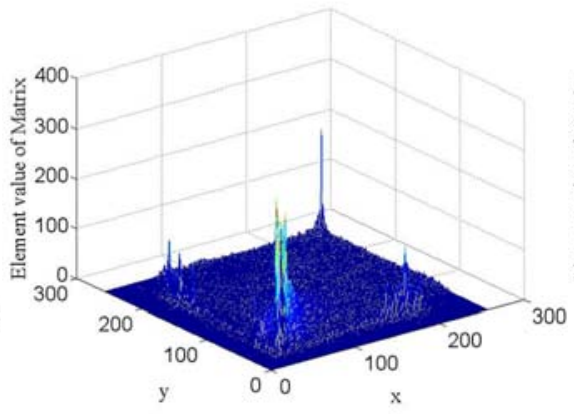

(e)

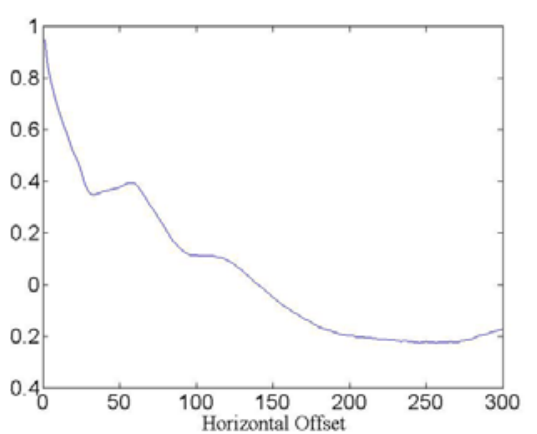

(c)

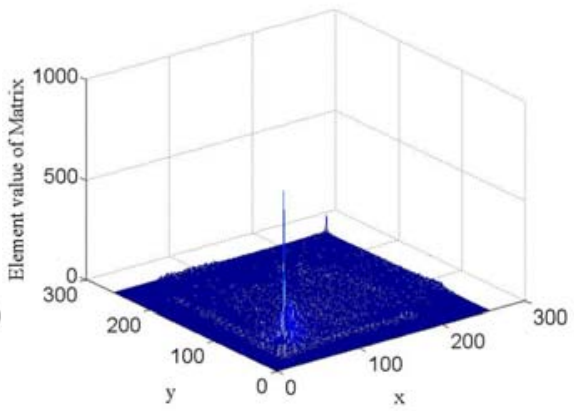

(f)

Fig.2. Experimental results on an oblique chessboard image. (a) Raw image, (b) Texture correlation as a function of offset in vertical direction, (c) Texture correlation as a function of offset in horizontal direction, (d) Texture correlation as a function of offsets in vertical direction and horizontal direction, (e) Co-occurrence matrix with the horizontal offset of one square, (f) Co-occurrence matrix with the horizontal offset of two squares.

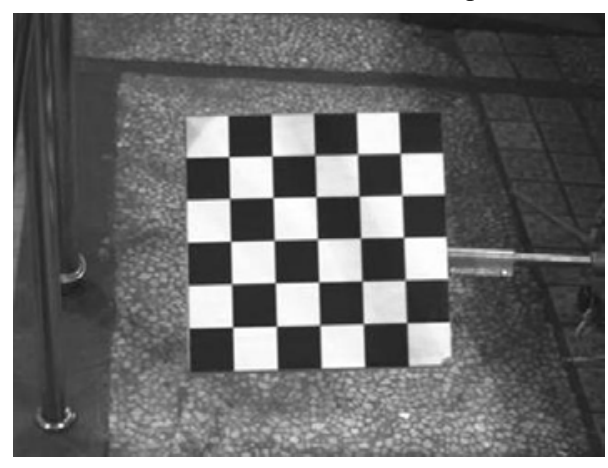

(a)

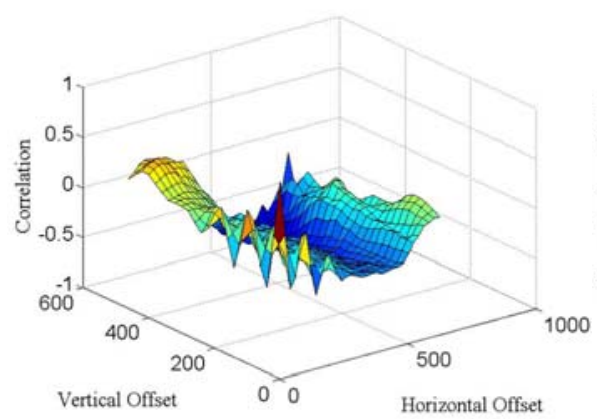

(d)

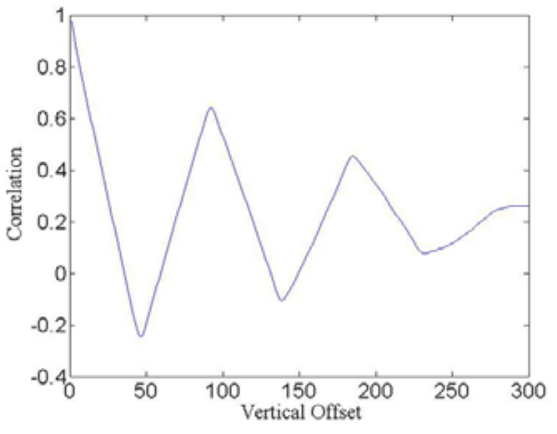

(b)

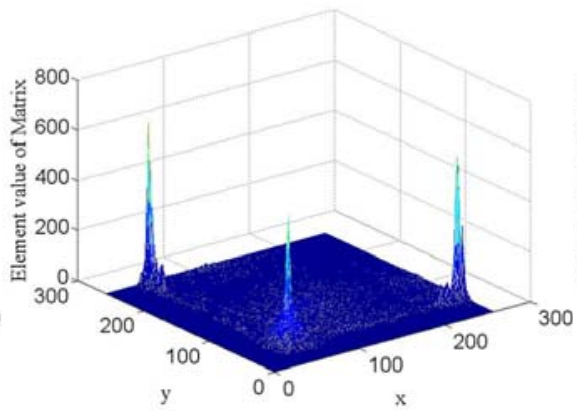

(e)

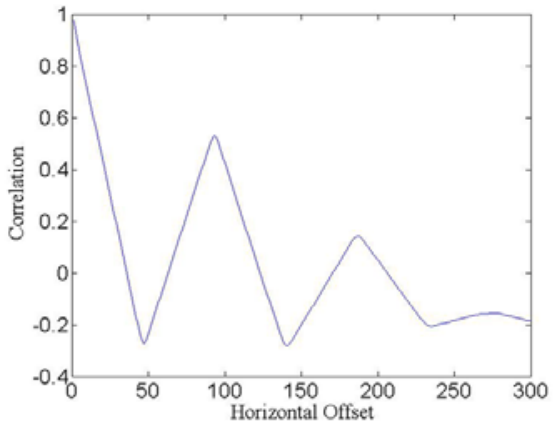

(c)

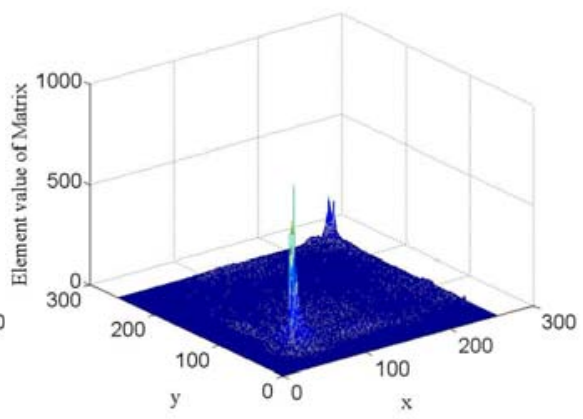

(f)

Fig.3. Experimental results on an orthographic chessboard image in large scale. (a) Raw image, (b) Texture correlation as a function of offset in vertical direction, (c) Texture correlation as a function of offset in horizontal direction, (d) Texture correlation as a function of offsets in vertical direction and horizontal direction, (e) Co-occurrence matrix with the horizontal offset of one square, (f) Cooccurrence matrix with the horizontal offset of two squares. 


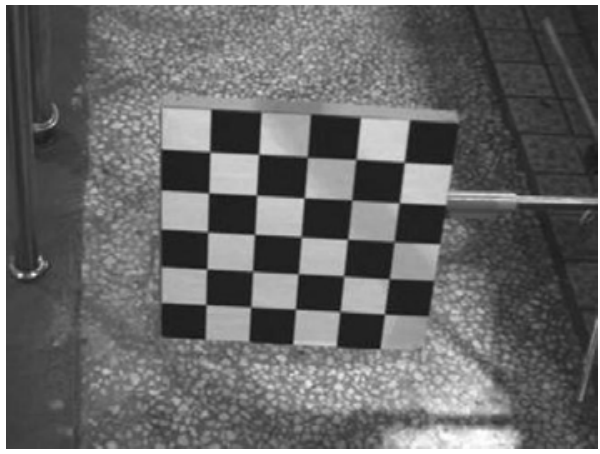

(a)

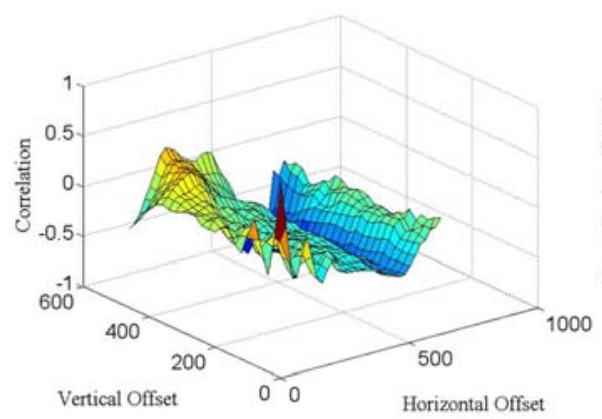

(d)

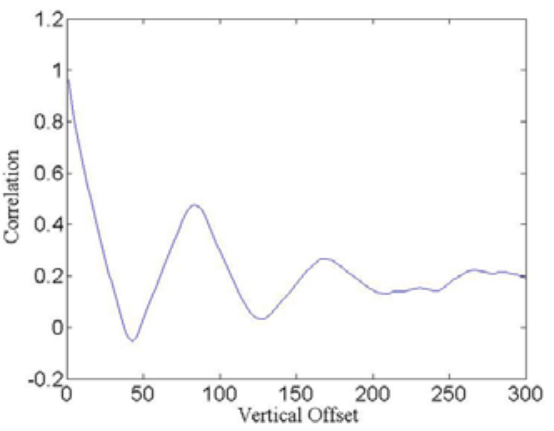

(b)

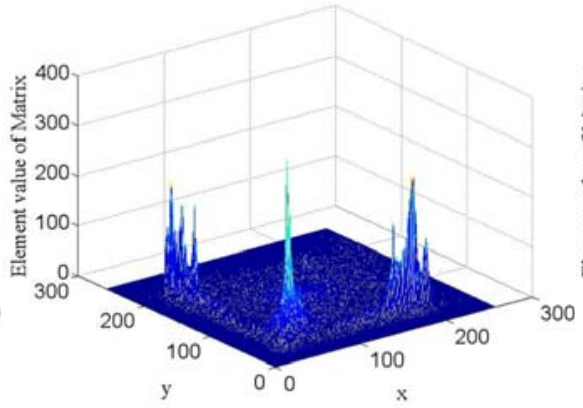

(e)

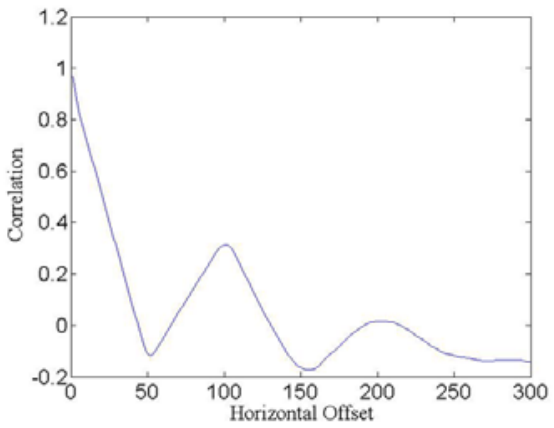

(c)

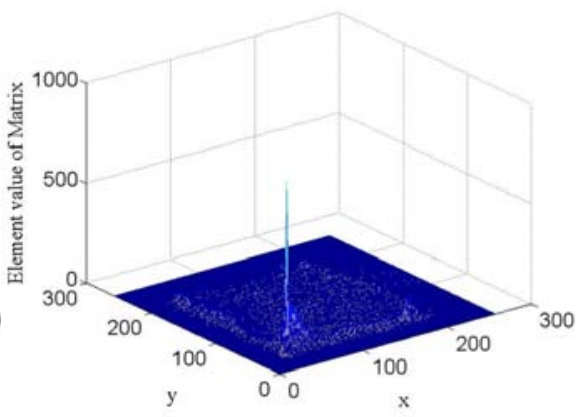

(f)

Fig.4. Experimental results on an orthographic chessboard image in large scale. (a) Raw image, (b) Texture correlation as a function of offset in vertical direction, (c) Texture correlation as a function of offset in horizontal direction, (d) Texture correlation as a function of offsets in vertical direction and horizontal direction, (e) Co-occurrence matrix with the horizontal offset of one square, (f) Cooccurrence matrix with the horizontal offset of two squares.

\section{CONCLUSIONS}

An approach of measuring the square size of the chess texture which is often adopted in a target board image in virtue of using gray-level co-occurrence matrix is employed to explore whether it is effective for different scale images.

According to the statistical performance of the chessboard on which white squares are separated by black squares in the same size accurately, the co-occurrence matrix with the parameters of offsets is computed on an image. To compare the repetition probability of the square texture in the image, the correlation of the co-occurrence matrices in which the offsets are parameterized is chosen as a ruler of measuring the squares. We concentrate on analyzing the tendency of the curves which is derived from the offsets varying in the range of half size of the image.

The experiments are executed on the condition that the offset varies in vertical and horizontal directions to obtain the distribution of texture invariance in different directions separately. As the repetition probability of the similar textures decreases with offset increasing, the correlation curves generally indicate a descending trend. Nevertheless, there are two obvious minimum and maximum in every experimental correlation curve. The first minimum of the horizontal curve describes the length of a square on the chessboard while the maximum in the vertical curve illustrates the width of a square. The experiment results of the length and width of the squares satisfy the manual measurement results. In summary, the method which utilizes the gray-level co-occurrence matrix for the chessboard image demonstrates that the proposed procedure is effective and appropriate for measuring the size of square.

\section{ACKNOWLEDGMENT}

The research is funded by Ph.D. Programs Foundation of Ministry of Education of China, under Grant No.20100061120067, Jilin Province Science Foundation for Youths, under Grant No.20100167, and China Postdoctoral Science Foundations, under Grant No.201104525 and No.20100471254.

\section{REFERENCES}

[1] De la Escalera, A., Armingol, J.M. (2010). Automatic chessboard detection for intrinsic and extrinsic camera parameter calibration. Sensors, 10 (3), 2027-2044.

[2] Mallon, J., Whelan, P.F. (2007). Calibration and removal of lateral chromatic aberration in images. Pattern Recognition Lett., 10 (3), 2027-2044.

[3] Zhao, Z.X., Wen, G.J. (2011). Ball's motion estimation using a line-scan camera. Measurement Science Review, 11 (6), 185-191.

[4] Brodic, D. (2011). Methodology for the evaluation of the algorithms for text line segmentation based on extended binary classification. Measurement Science Review, 11 (3), 71-78. 
[5] Xu, G., Li, X.T., Su, J., Pan, H.D., Tian, G.D. (2011). Precision evaluation of three-dimensional feature points measurement by binocular vision. J. Opt. Soc. Korea, 15 (1), 30-37.

[6] Tsai, C.H., Liou, C.S. (2001). Applying an on-line crack detection technique for laser cutting by controlled fracture. Int. J. Adv. Manuf. Technol., 18 (10), 724-730.

[7] Rosenfeld, A., Johnson, E. (1973). Angle detection on digital curves. IEEE Trans. Comput., 22 (9), 875-878.

[8] Freeman, H., Davis, L.S. (1977). A corner-finding algorithm for chain code curves. IEEE Trans. Comput., 26 (3), 297-303.

[9] Xiao, J.L., Xiang, Z.J., Wang, B., Liu, Y.C. (2011). Adaptive algorithm for corner detecting based on the degree of sharpness of the contour. Opt. Eng., 50 (4), 047008 .
[10] Ha, J.E. (2009). Automatic detection of chessboard and its applications. Opt. Eng., 48 (6), 067205.

[11] Qu, Z.G., Wang, P., Gao, Y.H., Wang, P. (2011). Randomized SUSAN edge detector. Opt. Eng., 50 (11), 110502.

[12] Lowe, D.G. (2004). Distinctive image features from scale-invariant keypoints. Int. J. Comput. Vision, 60 (2), 91-110.

[13] Harris, C., Stephens, M. (1998). A combined corner and edge detector. In Proceedings of the 4th Alvey Vision Conference (AVC88), 147-151.

[14] Haralick, R.M., Shanmugam, K., Dinstein, I. (1973). Textural features for image classification. IEEE Trans. Syst. Man Cybern., 3 (6), 610-621.

Received December 19, 2011. Accepted April 2, 2012. 